\title{
Physikalisch-chemische Untersuchungen über das Verhalten der harnsauren Salze in Lösungen.
}

\author{
Von
}

F. Gudzent, med. pract.

Mit 1 Kurvenzeichnung.

(Der Redaktion zugegangen am 13. Mai 1908.)

Durch W. His und Th. Paul') sind die ersten Daten gegeben worden, welche das Verständnis der Lösung harnsaurer Salze im menschlichen Harn auf einer exakten, physikalisch-chemischen Grundlage vorzubereiten geeignet sind. Die Arbeiten haben eine langdauernde Unterbrechung erlitten. Nachfolgende Untersuchung ist bestimmt, weitere Daten mit möglichster Exaktheit zu liefern und zwar zunächst über die Löslichkeit der primären harnsauren Salze des Natriums, Kaliums und Ammoniums bei $18^{\circ}$ und $37^{\circ}$.

Untersuchungen sind in früherer Zeit von Bentsch, Allar und Baumgarten ${ }^{2}$ ) und $\mathrm{Maly}^{3}$ ) angestellt worden (zusammengestellt in Beilsteins Handbuch der organischen Chemie 3. Aufl. 1903):

*Bei $100^{\circ}$ hat $1 \mathrm{Mol}$ saures harnsaures Natrium ${ }^{1 / 2} \mathrm{H}_{2} \mathrm{C}$ Krystallwasser. Es bildet sich beim Kochen von Harnsäurf mit Soda, Borax, Natriumphosphat oder Natriumacetat aus dem neutralen Salz durch Behandeln mit $\mathrm{CO}_{2}$. Es fällt als Krystallpulver aus. Ein Teil löst sich in 1100-1200 Teiler Wasser von $15^{\circ}$ und 123-125 Teilen siedenden Wassers Als Harnsediment erscheint es in amorphen Körnern. Aus de:

1) Diese Zeitschrift, 1900, Bd. XXXI.

2) Li ebigs Annalen d. Chem. u. Pharmac., Bd.LIV, S. 189 ; Bd. LXV S. $181 ;$ Bd. CXVII, S. 106.

3) Jahresbericht der Chemie, 1863. 
kalt gesättigten, wässerigen Lösung des Salzes fällt auf Zusatz von $\mathrm{Na}_{2} \mathrm{PO}_{4}, \mathrm{NaCl} \ldots \ldots$ ein Salz $\mathrm{NaC}_{5} \mathrm{H}_{3} \mathrm{~N}_{4} \mathrm{O}_{3}+1 \frac{1}{1 / 2} \mathrm{H}_{2} \mathrm{O}$ in Kugeln aus, welches bei $130^{\circ}$ noch $1 \mathrm{H}_{8} \mathrm{O}$ zurückbehält. Das neutrale Salz erscheint als $\mathrm{Na}_{2} \mathrm{C}_{5} \mathrm{H}_{2} \mathrm{~N}_{4} \mathrm{O}_{3}+\mathrm{H}_{2} \mathrm{O}$ in Warzenform. Es löst sich in 62 Teilen Wasser, wobei es aber zum Teil in saures Salz übergeht.

Das saure harnsaure Kalium erscheint als amorphe Masse oder in Körnern. Es löst sich in 700-800 Teilen Wasser von $20^{\circ}$, in 70-80 Teilen siedenden Wassers. Das neutrale Salz $\mathrm{K}_{2} \mathrm{C}_{5} \mathrm{H}_{2} \mathrm{~N}_{4} \mathrm{O}_{3}$ fällt in kleinen Nadeln aus. Es löst sich in 36 Teilen Wasser von $16^{\circ}$ unter Bildung von saurem Salz. Es reagiert stark alkalisch.

Ein neutrales Ammoniaksalz läßt sich nicht darstellen. Ein Teil des sauren Salzes löst sich in 1608 Teilen Wasser von $15^{\circ}$. $3 \mathrm{NH}_{3} 2 \mathrm{C}_{5} \mathrm{H}_{4} \mathrm{~N}_{4} \mathrm{O}_{3}$ scheidet sich aus der heiß gesättigten Lösung der Harnsäure in ammoniakalischem Wasser beim Stehen als amorphes Pulver ab. $4 \mathrm{NH}_{3} 3 \mathrm{C}_{3} \mathrm{H}_{4} \mathrm{~N}_{4} \mathrm{O}_{3}$ wird aus einer ammoniakalischen $50^{\circ}$ warmen Lösung von Harnsäure durch Alkohol in mikroskopischen Nadeln gefällt.»

Seit dieser Zeit sind Löslichkeitsbestimmungen an harnsauren Salzen nicht mehr vorgenommen worden. Da die Werte von Allan und Bentsch nach der Methodik ihrer Gewinnung mit Fehlern behaftet sein mußten, ergab sich für His und Paul das Bedürfnis, die Löslichkeitsbestimmung von neuem aufzunehmen. ${ }^{1}$ ) Ihre Resultate sind nicht ausführlich publiziert worden. Ich danke den Herren für die Erlaubnis, sie hier mitzuteilen.

Versuche von His and $\mathrm{Paul}$ über die Löslichkeit harnsaurer Salze.

Ausgangsmaterial durften nur möglichst reine und gut krystallisierte Salze sein.

$\mathrm{Da}$ die Harnsäure in wässeriger Lösung, namentlich in der Wärme ziemlich rasch zersetzt wird, versuchten wir, die Salze des Natriums, Kaliums und Ammoniums auf kaltem Wege zu gewinnen. Die Harnsäure wurde in Wasser aufgeschwemmt

1) Pharmaz. Zeit., 1900. 
und entweder die berechnete Menge kohlensaurer Alkalien zugesetzt oder die Lösung der Harnsäure im Überschuß des Alkali mit Kohlensäure behandelt. Indessen wurden auf diese Weise nur ausnahmsweise krystallinische Salze erhalten, meist amorphe oder aus amorphen und krystallinischen Teilen gemischte Fällungen, gleichviel, ob die Flüssigkeit in fortwährender Bewegung gehalten oder in völliger Ruhe nur an ihrer Oberfläche von einem Strom Kohlensäure getroffen wurde. Auch führten Versuche, die amorphen Massen mit Krystallen der primären harnsauren Alkalien zu impfen, zu keinem brauchbaren Produkt.

Die Anwendung der Wärme bei der Bereitung war unumgänglich zur sicheren Erlangung eines krystallinischen Produkts.

Am besten bewährte sich folgendes Verfahren:

*Zu zwei Mol stark verdünnter Kali- oder Natronlauge wurde ein Mol in Wasser suspendierte Harnsäure gefügt, filtriert, der klaren Lösung ca. 2 Mol Alkalibicarbonat zugefügt, nach 24 Stunden das amorphe Urat auf der Saugpumpe gesammelt, mit chloridhaltigem Wasser gewaschen, dann in viel siedendes Wasser unter Umrühren eingetragen (unter Zusatz von wenig Alkalichlorid, das die Bildung kolloidaler Lösung hindert), filtriert, das Filtrat an ruhigem Orte der Abkühlung überlassen. Auf diese Art erhält man das primäre Natriumurat in schönen mikroskopischen Nadeln. Sie enthalten 1 Molekül Krystallwasser, das bei $130^{\circ}$ nicht entweicht. Das primäre Kaliumsalz bildet ebensolche Nadeln, ist aber wasserfrei.

Das Ammonsalz wird erhalten, indem man zu viel siedendem Wasser Ammoniak setzt und allmählich eine wässerige Aufschwemmung von Harnsäure einträgt. Die ersten Portionen werden klar gelöst. Dann wird die Harnsäure umgesetzt in primäres Ammonsalz, das sofort schöne Nadeln bildet. Nach einigen Minuten wird abfiltriert und das Filtrat der Abkühlung überlassen, wobei feine, wasserfreie Nadeln ausfallen.

Die Reinheit der Salze wurde durch Bestimmung ihres Harnsäuregehaltes geprüft. Eine gewogene Menge wurde mit wenigen Kubikzentimetern Wasser und viel Salzsäure versetzt, nach 24 Stunden die ausgefallene Harnsäure gesammelt, mit 
Über das Verhalten der harnsauren Salze in Lösungen.

ganz wenig Wasser, Alkohol und Äther gewaschen, bei $60^{\circ}$ getrocknet.

Vier Bestimmungen ergaben für das Natriumsalz 81,04, $80,89,80,56,80,86 \%$ Harnsäure; berechnet sind für $\mathrm{C}_{5} \mathrm{H}_{3} \mathrm{~N}_{4} \mathrm{O}_{3}$ $+\mathrm{H}_{2} \mathrm{O} 80,77 \%$.

Zwei Bestimmungen des Kalisalzes ergaben 80,1 und $80,23 \%$, das wasserfreie Salz verlangt $81,55 \%$, das Salz mit 1 Molekül Krystallwasser 75,00\%.

Zwei Bestimmungen des Ammonsalzes ergaben 91,91 und $91,34 \%$, das wasserfreie Salz verlangt 90,81\%.

Die Löslichkeit der harnsauren Salze wurde bestimmt, indem ein Überschuß des Salzes mit gewogener Menge Wasser im Jenaer Kolben bei konstanter Temperatur verschiedene Zeit ( $t$ ) in beständiger Bewegung gehalten, der Rückstand im Goochschen Tiegel gesammelt, mit Alkohol und Äther gewaschen, bei $60^{\circ}$ getrocknet und gewogen wurde. In zwei Versuchen wurde die Lösung im Platintiegel eingedampft und der Rückstand gewogen.

A. Natronsalz. $\mathrm{C}_{3} \mathrm{H}_{3} \mathrm{~N}_{4} \mathrm{O}_{3} \mathrm{Na}+\mathrm{H}_{2} \mathrm{O}$.

Löslichkeit bei $18^{\circ}$.

\begin{tabular}{c|c|c|c|c}
\hline \hline & Nr. & $\begin{array}{c}\text { Gelöst in } \\
1 \text { l }\end{array}$ & $\begin{array}{c}\text { 1 Mol gelöst } \\
\text { in Litern (v) }\end{array}$ & $\begin{array}{c}\mathrm{t} \\
\text { Stunden }\end{array}$ \\
\hline \multirow{3}{*}{ Ungelöster Rückstand gewogen } & II a & 0,9258 & 224,6 & 21 \\
& IIb & 0,9178 & 226,6 & 27 \\
Abdampfrückstand der & III a & 0,8207 & 253,4 & 24 \\
Lösung gewogen & III b & 0,8100 & 256,8 & 48
\end{tabular}

In Versuch IIIa und $\mathrm{b}$ war der Abdampfrückstand frei von Krystallwasser. Die Berechnung auf krystallwasserhaltiges Salz ergibt:

\begin{tabular}{l|c|c|c|c}
\hline & Nr. & $\begin{array}{c}\text { Gelöst in } \\
11\end{array}$ & $\begin{array}{c}1 \text { Mol gelöst } \\
\text { in Litern (v) }\end{array}$ & $\begin{array}{c}t \\
\text { Stunden }\end{array}$ \\
\hline & III a & 0,8984 & 232,0 & 24 \\
& III b & 0,8867 & 230,7 & 48
\end{tabular}


Das Mittel aus II und III ergibt bei $18^{\circ}$ in einem Liter gelöst 0,9072

1 Mol gelöst in Liter 228,5.

Löslichkeit bei $37,5^{\circ}$.

Eine annähernde Bestimmung ergab in 11 gelöst 1,5946, 1 Mol gelöst in Liter 130,44.

B. Kaliumsalz. $\mathrm{C}_{5} \mathrm{H}_{3} \mathrm{~N}_{4} \mathrm{O}_{3} \mathrm{~K}$.

Löslichkeit bei $18^{\circ}$.

\begin{tabular}{|c|c|c|c|}
\hline & $\mathrm{Nr}$. & In 11 gelöst & $\begin{array}{c}1 \text { Mol gelöst } \\
\text { in Litern }\end{array}$ \\
\hline \multirow{2}{*}{ Abdampfungsrückstand des Filtrats } & I & 1,636 & 125,9 \\
\hline & \multirow[t]{2}{*}{ II } & 1,6454 & 125,26 \\
\hline Mittel . . . & & 6,641 & 125,7 \\
\hline
\end{tabular}

C. Ammoniumsalz. $\mathrm{C}_{5} \mathrm{H}_{3} \mathrm{~N}_{4} \mathrm{O}_{3} \mathrm{NH}_{4}$.

Löslichkeit bei $18^{\circ}$.

Die Löslichkeit wurde sowohl aus der Wägung des ungelöst gebliebenen Salzes als aus dem Abdampfrückstand bestimmt. Letzterer verliert etwas Ammonsalz und muß daher nach dem Abdampfen nochmals mit einigen Tropfen $\mathrm{NH}_{3}$ abgeraucht werden.»

\begin{tabular}{c|c|c|c}
\hline \hline & Nr. & In 1 l gelöst & $\begin{array}{c}\text { 1 Mól galöst } \\
\text { in Litern }\end{array}$ \\
\hline \begin{tabular}{c|c|c|c} 
Rückstand gewogen \\
Abdampfrückstand des Filtrats \\
gewogen
\end{tabular} & I & 0,2947 & 661,7 \\
I & II & 0,2990 & 652,2 \\
\cline { 2 - 3 } & & 0,2923 & 667,1 \\
\hline
\end{tabular}

Wo anderweitig sich nähere Angaben über das physikalisch-chemische Verhalten der harnsauren Salze finden, beziehen sie sich, soweit das beurteilt werden konnte, auf die Untersuchungen von Bentsch und Allan.

\section{Meine Löslichkeitsversuche.}

Die Harnsäure ist zweibasisch und demnach befähigt, primäre und sekundäre Salze zu bilden. Die sekundären Salze 
sind aber in wässeriger Lösung nicht beständig, sondern wandeln sich sofort in primäres Salz um. Das war zum Teil auch schon früher bekannt, die Erklärung gab jedoch erst die physikalische Chemie.

Wenn sekundäres Urat in Wasser aufgelöst wird, dissoziiert es stark in seine Ionen. Nun ist die Harnsäure eine Säure, deren primäres H-Ion schwach, deren sekundäres H-Ion aber kaum dissoziiert. Das Produkt aus der Konzentration der sekundären Urat-Anionen und der H-Kationen des ebenfalls dissoziierten Wassers wird infolge dessen zu groß, als daß beide so nebeneinander existieren können; es erfolgt die Reaktion:

$$
\mathrm{C}_{5} \mathrm{H}_{2} \overline{\overline{\mathrm{N}}} \mathrm{O}_{3}+\stackrel{+}{\mathrm{H}}=\mathrm{C}_{5} \mathrm{H}_{3} \overline{\mathrm{N}}_{4} \mathrm{O}_{3}
$$

Unter Hydrolyse des Wassers geht der Vorgang weiter, bis die Gleichungen

$$
\begin{aligned}
& \frac{\mathrm{CC}_{5} \mathrm{H}_{8} \mathrm{~N}_{4} \mathrm{O}_{8} \cdot \mathrm{CH}}{\mathrm{CC}_{5} \mathrm{H}_{8} \mathrm{~N}_{4} \mathrm{O}_{3}}=\mathrm{K}_{1}, \frac{\mathrm{CC}_{6} \mathrm{H}_{8} \mathrm{~N}_{4} \mathrm{O}_{8} \cdot \mathrm{CSalz} \text {-Kat. }}{\mathrm{CC}_{6} \mathrm{H}_{8} \mathrm{~N}_{4} \mathrm{O}_{8} \text { Salz-Kat. }}=\mathrm{K}_{2} \\
& \text { und } \mathrm{CH}_{\mathrm{H}} \cdot \mathrm{COH}=\mathrm{K}
\end{aligned}
$$

erfüllt sind.

Da in jedem Falle auf diese Weise das sekundäre Salz unter Zurücklassung von Salz-Kationen und Hydroxyl-Anionen in das primäre sich umwandeln muß, kann in wässerigen Lösungen stets nur eine Bestimmung der Löslichkeit primärer Salze möglich sein.

Die in der Literatur angegebenen Löslichkeitswerte sekundërer Urate in Wasser beruhen somit auf einem grundsätzlichen Irrtum der Untersucher.

Darstellung der harnsauren Salze.

Gemäß vorstehender Ausführungen kam für die vorliegenden Untersuchungen nur die Darstellung primärer Salze in Frage.

1. Primäres harnsaures Natrium.

Etwa 11 Wasser wird auf $40-50^{\circ}$ erwärmt, Natronlauge, dem Molengewicht der Harnsäuremenge entsprechend, zugesetzt, etwa $10 \mathrm{~g}$ einer von der Firma Merk in Darmstadt in 
sehr reinem Zustande bezogenen Harnsäure in Wasser aufgeschwemmt, langsam zugesetzt, die Auflösung durch Umrühren befördert und die nicht in Lösung gegangene Harnsäure schnell mittels Nutsche abfiltriert. In etwa einer halben Stunde beginnt im Filtrat der Ausfall des Salzes in mikroskopischen Nadeln. Nach etwa 24 Stunden wird das Salz abfiltriert, mit Aqua destillata bis zum Verschwinden der alkalischen Reaktion, dann mit Alkohol und Äther ausgewaschen, einige Tage im Exsikkator über Schwefelsäure, dann im Trockenofen bei $60^{\circ}$ bis zur Gewichtskonstanz (etwa 12-18 Stunden) getrocknet, gut gepulvert und in Trockengläsern aufbewahrt.

2. Primäres harnsaures Kalium.

Genau wie bei 1 ; nur ist es zweckmäßig, das Lösungswasser auf $70-80^{\circ} \mathrm{zu}$ erwärmen. Mikroskopische Nadeln.

3. Primäres harnsaures Ammonium.

Auf $10 \mathrm{~g}$ Harnsäure werden $3 \mathrm{l}$ Wasser zum Sieden erwärmt, überschüssiges Ammoniak zugesetzt, die aufgeschwemmte Harnsäure portionsweise zugesetzt, 2-3 Minuten im Sieden erhalten, dann rasch filtriert. Aus dem Filtrat fällt das Salz in mikroskopischen Nadeln.

Alle Salze, am leichtesten das Ammoniumurat, zersetzen sich beim Erwärmen über $60^{\circ}$, indem sie an der Oberfläche eine rötliche Färbung annehmen. Auch unterhalb $60^{\circ}$ bei längerer Erwärmung und selbst bei Zimmertemperatur innerhalb einiger Monate tritt die Zersetzung ebenfalls auf.

Der Krystallwassergehalt der Salze.

Eine gewogene Menge des Salzes wurde in $5 \mathrm{ccm}$ Wasser aufgeschwemmt, mit $2 \mathrm{ccm}$ Salzsäure versetzt, nach 24 Stunden die ausgefällte Harnsäure abfiltriert, gewaschen und bei $60^{\circ}$ getrocknet.

0,5134 g Natronsalz gab 0,4147 g Harnsäure $=80,77 \%$ Berechnet für $\mathrm{C}_{5} \mathrm{H}_{3} \mathrm{~N}_{4} \mathrm{O}_{3} \mathrm{Na}+\mathrm{H}_{2} \mathrm{O}=80,77 \%$

0,703 g Kaliumsalz gab 0,5659 g Harnsäure $=80,50 \%$

Berechnet für wasserfreies $\mathrm{Salz}=81,55 \%$ für $\mathrm{C}_{5} \mathrm{H}_{3} \mathrm{~N}_{4} \mathrm{O}_{3} \mathrm{~K}+\mathrm{H}_{2} \mathrm{O}=75,00 \%$

$0,5216 \mathrm{~g}$ Ammoniumsalz gab $0,4741 \mathrm{~g}$ Harnsäure $=90,89 \%$

Berechnet für wasserfreies Salz $=91,30 \%$ 
Das Natriumsalz krystallisiert also mit 1 Molekül Krystallwasser, das Kalium- und Ammoniumsalz wasserfrei.

$$
\text { Die Versuchsanordnung. }
$$

Bei den Löslichkeitsbestimmungen wurden folgende Bedingungen eingehalten:

1. Verwendung frisch bergestellten, in seiner rein weißen Farbe unveränderten reinen Salzes.

2. Verwendung möglichst reinen, kohlensäurefreien Wassers. Der Grad der Reinheit ist durch die elektrische Leitfähigkeit zu ermitteln.

3. Benutzung widerstandsfähiger Glasgeräte.

4. Die Lösung ist durch Schütteln festen Salzes mit Wasser bei der Versuchstemperatur herzustellen.

5. Die Gehaltsbestimmung der Lösung hat möglichst bald nach Erreichung des Sättigungspunktes zu geschehen.

6. Der Gehalt der Lösung ist durch Rückwägen des nicht gelösten Salzes zu bestimmen.

Die Forderung zu 1 ergibt sich aus früheren Erörterungen über die Herstellung der Salze (S. 156) und der Beobachtung, daß altes, in seiner Farbe verändertes Ammoniumurat unter sonst gleichen Bedingungen löslicher ist, als frisch hergestelltes Urat:

Ammoniumurat Nr. 44 (frisch hergestellt) löst sich $1: 2487$; Ammoniumurat Nr. 43 (mehrere Jahre alt, mit rötlicher Verfärbung) löst sich $1: 1834$.

Die Forderung zu 2 ergibt sich aus folgenden zwei Versuchsreihen:

\section{Versuchsreihe.}

Als Lösungswasser dient gewöhnliches destilliertes Wasser, das zu verschiedenen Zeiten aus der im Laboratorium befindlichen Flasche entnommen wurde und unter diesen Umständen in schwankender Menge $\mathrm{CO}_{2}$ und andere Stoffe enthält.

a) Ammoniumurat Nr. 42 löst sich 1:2202

b) 》 $\quad 41$ " $1: 2407$

a) Kaliumurat $\quad 21 \gg, 1: 591$

b) » $\quad 22$ » $1: 546$ 
II. Versuchsreihe.

Als Lösungswasser wird kohlensäurefreies Wasser von der Leitfähigkeit $0,87 \times 10^{-6}$ benutzt.

a) Ammoniumurat Nr. 42 löst sich $1: 2375$

b) 》 $\quad$ \% $\gg 1: 2388$

Die übrigen Versuchsbedingungen waren in beiden Reihen die gleichen. In der ersten Reihe schwankten die Löslichkeiten beträchtlich, in der zweiten bleiben sie fast konstant. Daraus geht deutlich der Einfluß verunreinigten Lösungswassers hervor.

$\mathrm{Zu}$ den Löslichkeitsversuchen wurde das von der Firma Kahlbaum gelieferte sogenannte Leitfähigkeitswasser benutzt, das nach 24 stündigem Durchleiten von kohlensäurefreier Luft eine Leitfähigkeit von $0,87 \times 10^{-6}$ hatte und diese fast genau, unter der von $\mathrm{His}$ und $\mathrm{Paul}$ angegebenen Aufbewahrungsmethode, ${ }^{1}$ ) innerhalb 5 Monaten beibehielt.

Die Notwendigkeit der Beachtung der Gesichtspunkte zu 3, 4 und 5 ergibt sich ohne weiteres aus den bereits zitierten Untersuchungen von His und Paul. Auch in der Technik der Untersuchung konnte ich mich im wesentlichen an das von diesen Autoren angegebene Verfahren anlehnen. In einen Erlenmeyerkolben aus Jenaer Glas von $200 \mathrm{ccm}$, vorher mit Wasserdampf gereinigt, wird eine bestimmte Uratmenge, ctwa $0 ; 5 \mathrm{~g}$, gebracht, eine gewogene Menge Leitfähigkeitswasser zugesetzt, der Kolben durch einen Gummistopfen und eine darüber sitzende Gummikappe fest verschlossen, eine bestimmte Zeit in einem Thermostaten $^{2}$ ) geschüttelt, die Flüssigkeit mittels Gooch schen Tiegels abgesaugt, der Rückstand mit geringen Mengen Alkohol und Äther gewaschen, zuerst über Schwefelsäure im Exsikkator, dann im Trockenofen bei $60^{\circ}$ bis zur Gewichtskonstanz getrocknet und durch Wiegen bestimmt.

Bei der Untersuchungstemperatur von $18^{\circ}$ war ein Einfluß der meist geringen Differenz dieser zwischen der Zimmertempe-

1) Diese Zeitschrift, Bd. XXXI, 1900.

2) Es war der von His und Paul benutzte Thermostat; cfr. His und Paul, Diese Zeitschrift, Bd. XXXI, 1900. 
ratur beim Absaugen der Lösung nicht zu erwarten, dagegen bei $37^{\circ}$.

Die Beobachtung ergab, daß bei einer Zimmertemperatur von etwa $18^{\circ}$ die $37^{\circ}$ warme Lösung abnahm

$$
\begin{aligned}
& \text { um } 1^{\circ} \text { in } 2 \text { Minuten, } \\
& \text { » } 2^{\circ} \text { » } 5 \text { » } \\
& \text { - } 3^{0} \text { » } 10 \text { » }
\end{aligned}
$$

Das Absaugen nahm etwa 3 Minuten in Anspruch. Die Abkühlung während dieser Zeit wurde durch Einhüllen des Schüttelkolbens in ein etwa $40^{\circ}$ warmes Tuch, durch Vorwärmen des Goochschen Tiegels und durch Eingießen von immer sehr kleinen Mengen Lösung in diesen sehr gut vermieden.

Die Feststellung des Sättigungszeitpunktes.

Es konnte die bekannte Tatsache bestätigt werden, daß die schwerer löslichen Körper schneller als die leichter löslichen ihren Sättigungspunkt erreichen. Die zeitlichen Verschiedenheiten traten allerdings nur bei der Untersuchungstemperatur von $18^{\circ}$ deutlich hervor, bei $37^{\circ}$ ließen sich diese mit meiner

\begin{tabular}{|c|c|c|c|c|c|}
\hline \multicolumn{3}{|c|}{ Urat } & \multicolumn{2}{|c|}{$\begin{array}{c}\text { Sättigungszeitpunkt } \\
\text { nach Beginn des Schüttelns }\end{array}$} & Löslichkeit \\
\hline & Bei $18^{\circ}$ & & & & \\
\hline Prim. & Ammoniu & arat & 15 & nuten & $1: 2415$ \\
\hline$\searrow$ & Natrium & , & 45 & , & $1: 1201$ \\
\hline$\triangleright$ & Kalium & , & 120 & , & $1: 653$ \\
\hline & Bei $37^{\circ}$ & & & & \\
\hline Prim. & Ammoniu & arat & 15 & , & $1: 1375$ \\
\hline$\triangleright$ & $>$ & & 30 & , & $1: 1392$ \\
\hline$\triangleright$ & Natrium & $y$ & 15 & , & $1: 667$ \\
\hline$\triangleright$ & > & & 45 & , & 1: 671 \\
\hline , & Kalium & , & 15 & , & 1: 374 \\
\hline 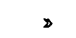 & , & & 30 & , & $1: 370$ \\
\hline
\end{tabular}
Methode wegen der sehr schnellen Sättigung nicht mehr ermitteln.

Alle Lösungen sind bei $37^{\circ}$ bereits nach 15 Minuten gesättigt. 
Die Abnahme der Löslichkeit mit der Zeit dis Schüttelns.

Bei meinen Versuchen trat eine Erscheinung zuta\&, die in bezug auf das Verhalten der primären Urate in wäseriger Lösung sehr bemerkenswert und bisher nirgends erwäht ist. Vergleicht man nämlich eine Reihe von Versuchen, diesonst unter den gleichen Bedingungen angestellt sind, so ergit sich die Tatsache, daß die Löslichkeit der Salze nach Errechung des Sättigkeitspunktes allmählich wieder abnimmt und de Geschwindigkeit dieser Abnahme immer geringer wird, je änger man das Salz schüttelt.

Es ergaben sich folgende Zahlen:

\begin{tabular}{|c|c|c|}
\hline Nr. der Versuchsreihe & Rotationsdauer & Löslichkeitsverältnis \\
\hline $\begin{array}{l}\text { Bei } 18^{\circ} \\
\text { Harnsaures Natrium Nr. } 3\end{array}$ & & \\
\hline VII & $\begin{array}{l}15 \text { Minuten } \\
45\end{array}$ & $\begin{array}{l}1: 1092 \\
1: 1073\end{array}$ \\
\hline I & 3 Stunden & $1: 1102$ \\
\hline II & 6 & $1: 1087$ \\
\hline VI & 12 & $1: 1107$ \\
\hline III & 24 & $\begin{array}{l}1: 1089 \\
1: 1099\end{array}$ \\
\hline IV & $48 \quad$ & $\begin{array}{l}1: 1132 \\
1: 1163\end{array}$ \\
\hline V & 72 & $1: 1113$ \\
\hline VIII & 8 Tage & $\begin{array}{l}1: 1151 \\
1: 1163\end{array}$ \\
\hline $\begin{array}{c}\text { Harnsaures Kalium Nr. } 22 \\
\text { VII }\end{array}$ & 15 Minuten & 1: 606 \\
\hline & $45 \quad$ & $\begin{array}{l}1: 599 \\
1: 576\end{array}$ \\
\hline I & 2 Stunden & 1: 546 \\
\hline II & 6 & $1: 559$ \\
\hline III & 14 & $1: 552$ \\
\hline $\mathrm{V}$ & 48 & $\begin{array}{l}1: 560 \\
1: 551\end{array}$ \\
\hline VI & 72 & $\begin{array}{l}\text { 1: } 572 \\
1: 566\end{array}$ \\
\hline VIII & 8 Tage & $1: 596$ \\
\hline
\end{tabular}




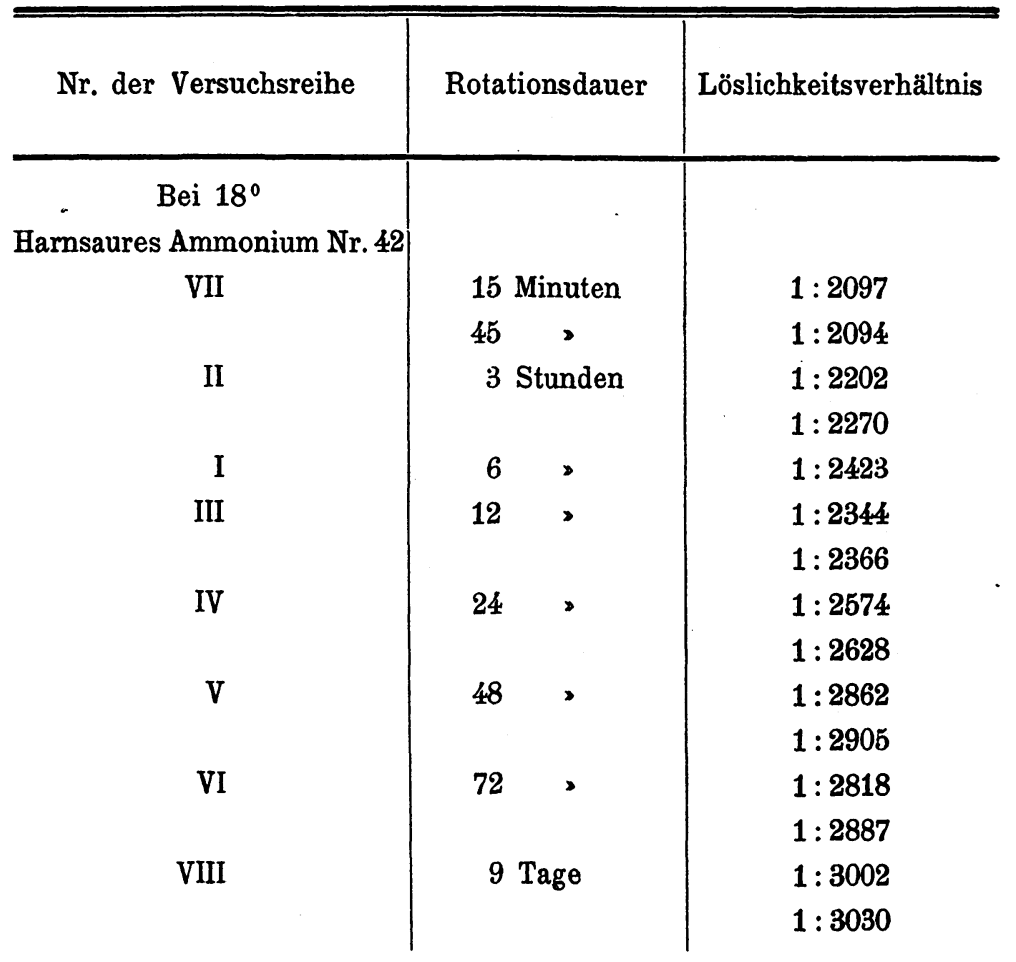

Folgende Kurven werden diese Löslichkeitsergebnisse besser veranschaulichen (siehe S. 162).

Vergleicht man zahlenmäßig die Werte der größten Löslichkeit mit den nach 8 bezw. 9 Tagen erhaltenen, so ergibt: sich eine Löslichkeitsabnahme um

$$
\begin{aligned}
8,4 \% & \text { beim Kaliumurat, } \\
7,8 \% & \text { » Natriumurat, } \\
31,0 \% & \text { Ammoniumurat. }
\end{aligned}
$$

Diese Werte entstammen Versuchsreihen, bei denen nicht einwandfreies Lösungswasser (ich benutzte gewöhnliches destilliertes Wasser aus der Laboratiumsflasche, das bei einer blinden Messung eine Leitfähigkeit von $5,1 \times 10^{-6}$ hatte) verwandt worden ist. 


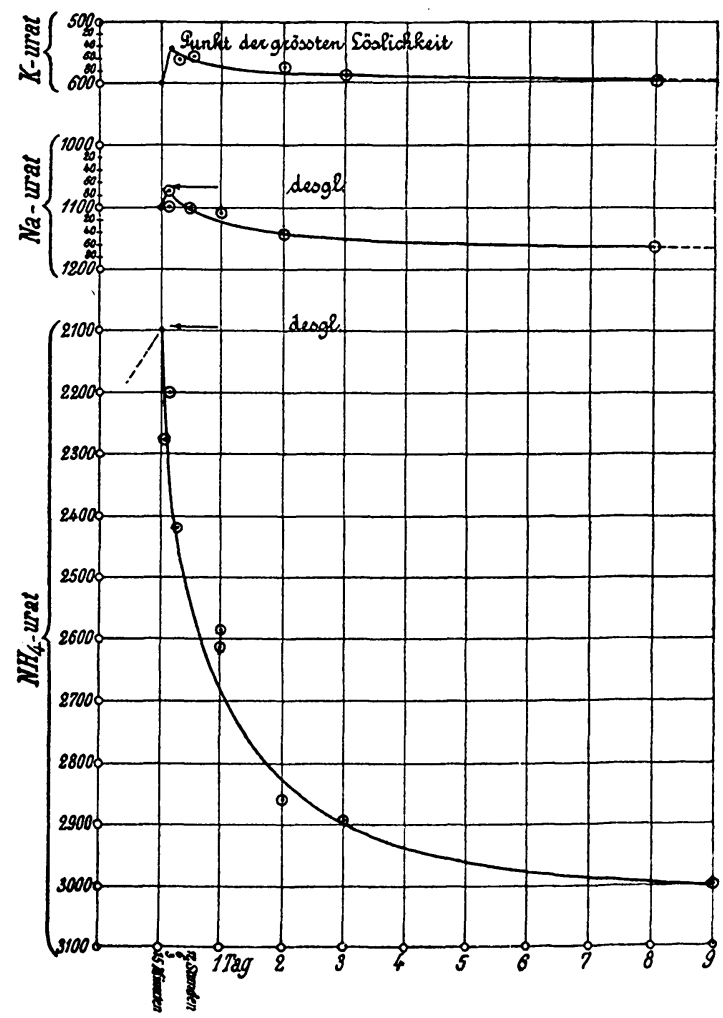

Doch ergaben die Versuche bei $37^{\circ}$, bei denen ein sehr reines Lösungswasser von der Leitfähigkeit $0,87 \times 10^{-6}$ benutzt wurde, fast dieselben Werte:

\begin{tabular}{c|c|c|c}
\hline & Rotationsdauer & $\begin{array}{r}\text { Löslichkeits- } \\
\text { verhältnis }\end{array}$ & $\begin{array}{c}\text { Abnahme } \\
\text { um }\end{array}$ \\
\hline Prim. Natriumurat & 15 Minuten & $1: 667$ & $4,7 \%$ \\
$>$. Kaliumurat & 8 Tage & $1: 699$ & \\
15 Minuten & $1: 374$ & $7,0 \%$ \\
, Ammoniumurat & 15 Minuten & $1: 1273$ & $31,7 \%$ \\
, & 8 Tage & $1: 1863$ &
\end{tabular}


Durch dieses Ergebnis wird zugleich der Gedanke an die Möglichkeit irgend eines Fehlers in der Versuchsanordnung ausgeschlossen.

Beim Ammoniumurat lassen die Zahlen und die Kurven kaum einen Zweifel an der Tatsache der Löslichkeitsabnahme $\mathrm{zu}$ : beim Natrium- und Kaliumurat dagegen läßt sich einwenden, daß die Veränderlichkeit der Löslichkeitswerte innerbalb der Fehlergrenzen liegen könnte.

Stellt man die Fehlergrenzen a (Differenzen der Grenzwerte nach kurzer Zeit) der Löslichkeitsabnahme $b$ innerhalb langer Zeit gegenüber, so zeigt sich, daß die letztere durchweg erheblich größer ist.

\begin{tabular}{|c|c|c|c|}
\hline & Urat & a $10^{2}$ & b $10^{2}$ \\
\hline \multirow[t]{3}{*}{$18^{\circ}$} & Natrium & 4 & 7 \\
\hline & Kalium & 8 & 15 \\
\hline & Ammonium & 1,5 & 15 \\
\hline \multirow[t]{3}{*}{$37^{\circ}$} & Natrium & 6 & 7 \\
\hline & Kalium & 10 & 18 \\
\hline & Ammonium & 5 & 25 \\
\hline
\end{tabular}

Auch zeigt das Kurvenbild (S. 162) eine trotz der mäßigen Krümmung der Kurve anfangs schnellere, später langsamere, aber entschiedene Tendenz zum Sinken.

Am ausgesprochensten tritt dieses beim Ammoniumsalz hervor. Hier läßt sich mit einem Blick die Löslichkeitsabnahme und die Veränderung der Geschwindigkeit in zweifelsfreier Weise übersehen.

Bei allen drei Uraten besteht also neben dem Lösungsmaximum ein Lösungsminimum. Da diese Erscheinung gerade für das Verhalten der Salze im Organismus von besonderer Bedeutung sein dürfte, werde ich in weiteren Untersuchungen dieses Lösungsminimum festzustellen versuchen.

Aus den Versuchen geht schließlich noch hervor, daß

1. die Tendenz zur Löslichkeitsabnahme sowohl bei $18^{\circ}$ wie bei $37^{\circ}$ beim Natrium- und Kaliumurat annähernd gleich groß, beim Ammoniumurat aber $4-5 \mathrm{mal}$ größer ist und 
2. die Steigerung der Temperatur von 18 auf $37^{\circ}$ auf diese Tendenzen keinen Einfluß hat.

Die Gründe der Löslichkeitsabnahme.

His und Paul haben festgestellt, ${ }^{1}$ ) daß, wenn Kohlensäure in eine wässerige Aufschwemmung von primärem Natriumurat eingeleitet wird, sich Harnsäure ausscheidet, obwohl diese eine größere Dissoziationskonstante als Kohlensäure hat. Der Vorgang findet seine Erklärung in der verschieden starken Löslichkeit der Harnsäure und Kohlensäure. 1 Mol Harnsäure löst sich erst in $6636 \mathrm{l}$ Wasser, $1 \mathrm{Mol}$ Kohlensäure dagegen schon in $44 \mathrm{l}$. Die Konzentration der H-Ionen in einer gesättigten Kohlensäurelösung ist daher im Verhältnis zu derjenigen in einer gesättigten Harnsäurelösung und mit Rücksicht darauf, daß die Dissoziation der Harnsäure durch die in Lösung befindlichen primären Harnsäureanionen des Natriumsalzes bedeutend zurückgedrängt wird, groß genug, um die Harnsäure in fester Form auszuscheiden.

$\mathrm{Da}$ nach His und $\mathrm{Paul}^{2}$ ) gewöhnliches, ohne besondere Vorsicht aufbewahrtes, destilliertes Wasser Kohlensäure in einer Konzentration von $1 \mathrm{Mol}$ in $1200 \mathrm{l}$ enthalten kann, diese Konzentration demnach etwa die fünffache der Harnsäure beträgt, war zu entscheiden, ob die in meinem nicht einwandfreien Lösungswasser enthaltene Kohlensäure das primäre Salz zersetzte und so die Löslichkeit zu klein erscheinen ließ.

Die Versuche auf Seite 162 und die folgenden Versuche sind mit einem kohlensäurefreien Wasser von der Leitfähigkeit $0,87 \times 10^{-6}$ angestellt. Ein Einfluß der Kohlensäure läßt sich aber aus ihnen nicht konstatieren.

Ammoniumurat, 15 Minuten geschüttelt, löst sich $1: 2375$ 48 Stunden » $1: 3085$

Es sind auch niemals im Bodenkörper, trotz eifrigen Suchens, Harnsäurekrystalle, die an ihrer Tafel- und Wetzsteinform leicht $\mathrm{zu}$ erkennen sind, gefunden worden.

1) Pharmac. Zeitung, 1900.

2) Diese Zeitschrift, Bd. XXXI, 1900. 
Es war weiterhin an einen etwaigen Einfluß der chemisch wirkenden Strahlen des Lichtes zu denken. Doch zeigt der hierunter aufgeführte Versuch, bei dem ein Schüttelkolben vollständig von allen Lichtstrahlen abgeschlossen in einem Säckchen von schwarzem Tuch sich befand, daß dieser Einfluß nicht vorhanden sein kann.

\begin{tabular}{ll|l|l}
\hline & & $\begin{array}{c}\text { Rotations- } \\
\text { dauer }\end{array}$ & Löslichkeit \\
\hline Ammoniumurat Nr. 42. & $\begin{array}{l}\text { Ohne Lichtabschl. } \\
\text { mit }\end{array}$ & $\begin{array}{l}48 \text { Stunden } \\
48,\end{array}$ & $\begin{array}{l}1: 3013 \\
1: 3085\end{array}$
\end{tabular}

Da die harnsauren Salze aus einer schwachen Säure und einer starken Base bestehen, könnte man an eine hydrolytische Spaltung denken, durch welche freie Harnsäure gebildet und infolge ihrer Schwerlöslichkeit ausgeschieden werden könnte. Bei manchen anorganischen Salzen sind derartige Ausfällungen gut zu beobachten.

Die hydrolytische Spaltung eines Urats läßt sich ausdrücken durch die Gleichung:

$$
\mathrm{C}_{5} \mathrm{H}_{3} \mathrm{~N}_{4} \mathrm{O}_{3}^{-}+\mathrm{H}_{2} \mathrm{O}=\mathrm{C}_{3} \mathrm{H}_{3} \mathrm{~N}_{4} \mathrm{O}_{3} \mathrm{H}+\mathrm{OH}^{-} \text {. }
$$

Nach Ablauf der Reaktion müssen dann in der Lösung folgende Gleichgewichte bestehen:

$$
\begin{aligned}
\frac{\mathrm{CH}^{+} \cdot \mathrm{CC}_{5} \mathrm{H}_{8} \mathrm{~N}_{4} \mathrm{O}_{3}^{-}}{\mathrm{CC}_{5} \mathrm{H}_{8} \mathrm{~N}_{4} \mathrm{O}_{3} \mathrm{H}} & =\mathrm{K}_{1} \\
\mathrm{CH}_{\mathrm{H}} \cdot \mathrm{COH} & =\mathrm{K}
\end{aligned}
$$

Durch Division ergibt sich:

$$
\frac{\mathrm{CoH} \cdot \mathrm{CC}_{5} \mathrm{H}_{8} \mathrm{~N}_{4} \mathrm{O}_{3} \mathrm{H}}{\mathrm{CC}_{5} \mathrm{H}_{8} \mathrm{~N}_{4} \mathrm{O}_{3}-}=\frac{\mathrm{K}}{\mathrm{K}_{1}} \text { oder } \frac{\mathrm{C}^{2} \mathrm{OH}}{\mathrm{C}_{5} \mathrm{H}_{8} \mathrm{~N}_{4} \mathrm{O}_{3}-}=\frac{\mathrm{K}}{\mathrm{K}_{1}} \text {, }
$$

denn

\section{$\mathrm{COH}$ und $\mathrm{CC}_{5} \mathrm{H}_{8} \mathrm{~N}_{4} \mathrm{O}_{3} \mathrm{H}$}

sind nach der obigen Reaktionsgleichung einander gleich.

Der Grad der Hydrolyse wird gegeben durch den Wert von $\mathrm{CoH}$. Es ist also: 


$$
\mathrm{CoH}=\sqrt{\frac{\mathrm{K} \cdot \mathrm{CC}_{5} \mathrm{H}_{8} \mathrm{~N}_{4} \mathrm{O}_{3}}{\mathrm{~K}_{1}}}
$$

Es ist nun:

$$
\begin{aligned}
& \mathrm{K}=0,64 \times 10^{-14} \\
& \left.\mathrm{~K}_{1}=0,151 \times 10^{-3}(\text { nach His und } \mathrm{P} \varepsilon u l)^{1}\right) .
\end{aligned}
$$

Den Wert für $\mathrm{CC}_{5} \mathrm{H}_{3} \mathrm{~N}_{4} \mathrm{O}_{3}$ kann man mit Rücksiclt auf die fast vollständige Dissoziation und geringe hydrolyische Spaltung der Urate ihrer angewandten Konzentration deichsetzen. In den bei meinen Untersuchungen vorliegenden gesättigten Lösungen wäre dann

$\mathrm{CC}_{6} \mathrm{H}_{s} \mathrm{~N}_{4} \mathrm{O}_{3} \cdot$ CUrat-Kation $=\mathrm{L}$ (Löslichkeitsproduk) und demnach

$$
\mathrm{CC}_{5} \mathrm{H}_{8} \mathrm{~N}_{4} \mathrm{O}_{8}=\sqrt{\mathrm{L}}
$$

So kommen wir zu folgendem Endausdruck:

$$
\mathrm{COH}=\sqrt{\frac{\mathrm{K}}{\mathrm{K}_{\mathrm{i}}} \sqrt{\mathrm{L}}}
$$

Beim Kaliumurat ist

$$
\sqrt{\mathrm{L}}=\sqrt{\left(\frac{1}{13 \tilde{5}}\right)^{2}}=\frac{1}{135}
$$

bei $18^{\circ}$, da $1 \mathrm{Mol}$ in $135 \mathrm{l}$ sich löst. Setzt man nun in di Endgleichung die Zahlenwerte ein, so ist

$$
\mathrm{CoH}=\sqrt{\frac{0,64 \times 10^{-14}}{0,151 \times 10^{-3} \cdot 135}}=5,6 \times 10^{-7}
$$

das heißt also, in einem Liter einer gesättigten Kaliunuratlösung sind bei $18^{0} 5,6 \times 10^{-7} \mathrm{Mol}$ hydrolytisch gespalter, oder da ja

$$
\mathrm{COH}=\mathrm{CC}_{5} \mathrm{H}_{3} \mathrm{~N}_{4} \mathrm{O}_{3} \mathrm{H}
$$

ist, in ebensoviel Harnsäure umgewandelt.

Nach His und $\mathrm{Paul}^{2}$ ) löst sich 1 Mol Harnsäure in $3636 \mathrm{l}$, $5,6 \times 10^{-7} \mathrm{Mol}$ also in $0,003 \mathrm{l}$, die gerade genügen, im das Ausfallen der Harnsäure zu verhindern.

1) Diese Zeitschrift, Bd. XXXI, 1900.

2) Diese Zeitschrift, Bd. XXXI, 1900. 
Über das Verhalten der harnsauren Salze in Lösungen.

Diese Ableitung gilt nur für die Salze der starken Basen Kalium und Natrium.

Für das Ammoniumurat, das Salz der schwachen Base, gilt nach Van't Hoff ${ }^{1}$ ) folgende Beziehung:

$$
\begin{gathered}
\frac{\mathrm{C}_{\text {Base }} \cdot \mathrm{CSSäure}^{2}}{\mathrm{C}_{\text {Salz }}}=\frac{\mathrm{K}}{\mathrm{K}_{1} \mathrm{~K}_{2}} \\
\mathrm{~K}=\mathrm{CoH}_{\mathrm{CH}}=0,64 \cdot 10^{-14}, \\
\left.\mathrm{~K}_{1}=\frac{\mathrm{CNH}_{4} \cdot \mathrm{COH}}{\mathrm{CNH}_{4} \mathrm{OH}}=1,8 \cdot 10^{-5}{ }^{2}\right)
\end{gathered}
$$

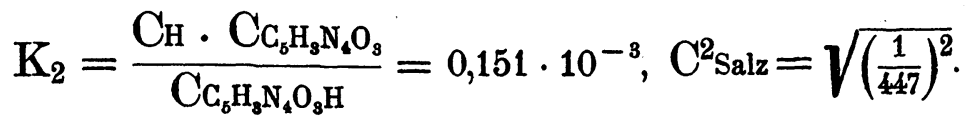

Dann ist:

$$
\begin{aligned}
\text { Case }_{\text {Bäure }} & =\sqrt{\frac{0,64 \cdot 10^{-14}}{1,8 \cdot 10^{-5} \cdot 0,151 \cdot 10^{-3}}\left(\frac{1}{447}\right)^{2}} \\
& =\frac{34 \cdot 10^{-7}}{\mathrm{C}_{\text {Base }}} \\
\text { Hydrolysengrad } & =\frac{34 \cdot 10^{-7}}{2,24 \cdot 10^{-3}} \\
& \left.=\frac{1,5 \cdot 10^{-8}}{\mathrm{C}_{\text {Salz }}}=0,15 \% 0^{8}\right) \\
\mathrm{C}_{1} \cdot \mathrm{CNH}_{4} \mathrm{OH} & =\frac{1,8 \cdot 10^{-5} \cdot 34 \cdot 10^{-7}}{\mathrm{CNH}_{4}} \\
& =0,24 \cdot 10^{-3}
\end{aligned}
$$

Da der Neutralpunkt

$$
\left(\mathrm{CoH}=\mathrm{CH}_{\mathrm{H}}\right)=0,8 \cdot 10^{-7}
$$

ist, wird die Ammoniumuratlösung im Gegensatz zu den beiden anderen Salzen, bei denen der Neutralpunkt unterhalb von $\mathrm{CoH}$ liegt und so eine schwach alkalische Reaktion bedingt, schwach sauer reagieren.

1) Van't Hoff, Vorlesungen über Bildung und Spaltung von Doppelsalzen, S. 125 u. 130.

2) Abegg, Handbuch der anorganischen Chemie, Bd. III, 3, S. 74, 1907.

8) Ist nach der Theorie von der Verdünnung unabhängig. 
Die gebildete Harnsäure von $34 \cdot 10^{-7}$ Mol bedarf zur Lösung 0,024 1 .

Da allen Berechnungen immer 11 Lösungswasser zugrunde liegt, kann die Hydrolyse nicht Ursache der Löslichkeitsverminderung sein.

Weil die Kenntnis des Hydrolysengrades der Urate sowie die $\mathrm{OH}-\mathrm{Konzentration}$ für mancherlei Zwecke von Interesse ist, seien hierunter die Werte, bezogen auf 11 einer gesättigten Lösung (ausgedrückt in Bruchteilen der Normallösung), zusammengestellt:

$18^{\circ}$.

$1 / 135$ (0,0074) -norm. Kaliumurat $\mathrm{CoH}=5,6 \times 10^{-7}$,

hydrolysiert $0,0076 \%$.

${ }^{3} / 250(0,004)$-norm. Natriumurat $\mathrm{COH}=4,12 \times 10^{-7}$,

hydrolysiert $0,001 \%$.

1/44i (0,002) -norm. Ammoniumurat

$$
\mathrm{COH}=0,27 \times 10^{-7}
$$

CHarnsäure $=34 \times 10^{-7}$ hydrolysiert $0,15 \%$.

Für $37^{\circ}$ lassen sich exakte Werte zunächst nicht geben, weil die Konstanten noch unbekannt sind. Doch läßt sich mit Sicherheit sagen, daß auch bei dieser Temperatur die Hydrolyse auf die Löslichkeitsabnahme ohne Einfluß ist.

Nach Ostwald ${ }^{1}$ ) tritt eine Änderung der Löslichkeit ein, wenn der gelöste Stoff in mehreren Modifikationen, also einer krystallinischen und einer amorphen, vorkommt und in der Lösung aus der einen in die andere Form übergeht. Ich habe aber mit besonderer Sorgfalt durch mikroskopische Untersuchungen darauf geachtet, daß die verwendeten Salze durchweg krystallinisch, ohne Einschluß amorpher Beimengungen, waren.

Bei der Prüfung der Dissoziationskonstante starker Elektrolyte, also insbesondere Salze, ergaben sich Anomalien für den Ausdruck des Massenwirkungsgesetzes $\frac{\alpha^{2} \cdot c}{1-\alpha}$ insofern, als dessen Werte für die verschiedenen Konzentrationen c erheb-

1) Lehrbuch der allgem. Chemie, 2. A., 1903. 
lich von der Forderung der Konstanz abweichen. Da diese Wertveränderungen in gewisser Weise den von mir gefundenen Löslichkeitsveränderungen analog sind, könnten einige chemische Tatsachen, Selbstkomplexbildung und Hydratation der Ionen, die zur Erklärung der Veränderlichkeit der Dissoziationskonstante herangezogen sind, ${ }^{1}$ ) auch für die Löslichkeitsveränderung in Betracht kommen.

Die Selbstkomplexbildung ${ }^{2}$ ) ist eine Anlagerung undissoziierter Molekeln des Elektrolyten an eine seiner Ionenarten und somit eine Verringerung der Zahl selbständiger Molekeln. In dem Falle gesättigter Lösungen müßte also zur Aufrechterhaltung des Löslichkeitsprodukts :

$$
\text { CAnion } \cdot \text { CKation }=\mathrm{L}
$$

neues Salz gelöst, die Löslichkeit also erhöht werden. Diese Vorstellung ist deswegen hier nicht anwendbar.

Die Hydratation ${ }^{3}$ ) besteht darin, daß die Ionen Komplexe mit dem Lösungsmittel bilden. Mit jedem Mol eines sich bildenden Komplexes müßte also mindestens $1 \mathrm{Mol}$ Lösungsmittel verschwinden. Unter der Voraussetzung, daß sonst keinerlei Veränderungen in dem System erfolgen, muß bei gesättigten Lösungen der abnehmenden Menge des Lösungsmittels entsprechend Salz ausfallen und so die Löslichkeit abnehmen.

$1 \mathrm{Mol}$ Ammoniumurat löst sich in $447 \mathrm{l}$, also in $24834 \mathrm{Mol}$ Wasser. In 1 Mol lösen sich demnach $\frac{186,2}{24834}=0,00749 \mathrm{~g}$ Urat; d. h. also für je $1 \mathrm{Mol}$ Wasser, das verschwindet, können $0,00749 \mathrm{~g}$ Salz weniger gelöst werden. In diesem Sinne gedeutet sind nun nach Versuch VII und VIII 0,4768 - 0,3295 =0,1473 g aus der Lösung wieder verschwunden, demnach $\frac{0,1473}{0,00749} \mathrm{Mol}$ $=197 \mathrm{Mol}=3546 \mathrm{~g}$ Wasser zur Hydratation verbraucht. Da

1) Abegg, Theorie der elektrolytischen Dissoziation.

2) Hittdorf, Pogg. Ann., S. 106, 385 und 546 (1859); Bredig, Zeitschr. f. physik. Chem., Bd. XIII, S. 262 (1894); Noyes, Ibid., Bd. XXXVI, S. 63 (1901); Steele, Ibid., Bd. XL, S. 722 (1902).

3) W. Bilz, Zeitschr. f. physik. Chem., Bd. XL, S. 217 (1902). 
die vorhandene Menge des Lösungswassers aber nur etwa $180 \mathrm{~g}$ betrug, ist erwiesen, daß hier die Hydratation zur Erklärung der Löslichkeitsabnahme nicht herangezogen werden kann.

In analoger Weise ermittelt, würde bei den beiden andern Uraten die Hydratation etwa $90 \mathrm{~g}$, gleich der Hälfte des vorhandenen Lösungswassers, betragen und an sich wohl möglich sein; doch ist einerseits eine so weitgehende Hydratation noch nirgends beobachtet und im höchsten Grade unwahrscheinlich, andererseits die Ausnahmestellung des Ammoniumurats in keiner Weise zu rechtfertigen. Es muß demnach auch hier eine Deutung der Löslichkeitsabnahme in diesem Sinne abgelehnt werden.

Die Beobachtung, daß einige anorganische Salze ihren Krystallwassergehalt ändern, wenn die Temperatur, bei der sie sich gebildet haben, geändert wird, führt zu der Überlegung, daß die Urate, die sich ja bei weit höheren als den Versuchstemperaturen gebildet haben, in analoger Weise sich in Salze mit höherem Krystallwassergehalt umwandeln, den Rückstand also an Gewicht vermehren und so eine Löslichkeitsabnahme vortäuschen könnten. In diesem Sinne gedeutet, müßte also $1 \mathrm{Mol}$ Ammoniumurat etwa $5 \mathrm{Mol} \mathrm{H}_{2} \mathrm{O}, 1 \mathrm{Mol}$ Natrium- und Kaliumurat je $1 \mathrm{Mol} \mathrm{H}_{2} \mathrm{O}$ addiert haben. Nun ist aber die Löslichkeitsabnahme bei $37^{\circ}$ der bei $18^{\circ}$ fast gleich, d. h. also in diesem Falle, auch das Maß der Krystallwasserbildung. Da derartige beobachtete Umwandlungen bei anderen Temperaturen aber auch andere Werte haben, ist eine Erklärung der Löslichkeitsabnahme bei den Uraten in diesem Sinne nicht zu geben.

Es wäre möglich, daß die Urate schwerer lösliche Komplexverbindungen bildeten, wobei an die von Sir W. Roberts ${ }^{1}$ ) genauer studierten Quadriurate zu denken wäre. Indessen sind diese in rein wässeriger Lösung nicht haltbar, außerdem amorph und an ihrer mikroskopischen Gestalt kenntlich. Der Rückstand bei meinen Löslichkeitsversuchen enthielt indessen niemals die von Roberts beschriebenen kugeligen Gebilde.

1) Sir W. Roberts, Uric Acid, Gravel and Gout, London 1892. 
Von Ostwald ${ }^{1}$ ) und G. A. Hulett ${ }^{2}$ ) ist nachgewiesen, daß Substanzen unter $2 \%$ Löslichkeit infolge Beziehungen der Oberflächenenergie zur Korngröße ihre Löslichkeit merklich vermindern, wenn sie längere Zeit mit dem Lösungsmittel in Berührung bleiben.

Diese Autoren folgern aus der Annahme, daß der Lösungsdruck eines Körpers und demnach seine wirkliche Löslichkeit eine Funktion der Oberflächenenergie ist, die Löslichkeit mit dem Abnehmen dieser, also mit dem Größerwerden der einzelnen Teilchen, abnimmt und umgekehrt.

Nun ist in einem heterogenen System, gebildet aus einer Lösung in Berührung mit einer Menge verschieden großer Teilchen einer Substanz, die Lösung in bezug auf die kleinsten Teilcken gesättigt, in bezug auf die größten aber übersättigt; diese müssen wachsen und eine Verminderung der Konzentration der Lösung verursachen. Dadurch wird wieder das Gleichgewicht mit den kleinen Teilchen zerstört, und diese werden aufgelöst. So gehen die Umformungen weiter, bis die Oberflächenenergie ein bestimmtes Minimum erreicht hat, denn nur dann ist das System in Gleichgewicht.

Durch entsprechende Versuchsanordnungen konnte Hulett zeigen, daß die Löslichkeitsabnahme innerhalb 9-10 Tagen bei Gips $20 \%$, beim Baryumsulfat sogar $80 \%$ betrug.

Diese Vorstellung von der Löslichkeitsabnahme läßt sich im allgemeinen gut auf die Löslichkeitsabnahme der Urate übertragen. Schwerlöslichkeit, Kurvenbild, Beobachtungszeit, Gleichheit des Vorganges bei $18^{\circ}$ und $37^{\circ}$, die große Wahrscheinlichkeit eines Löslichkeitsminimums sprechen durchaus dafür. Doch ergeben andere Beobachtungen und Überlegungen recht schwer wiegende Bedenken.

Je schwerer ein Salz sich löst, desto größer wird, wie das Beispiel von Gips- und Baryumsulfat zeigt, die Löslichkeitsabnahme sein.

Nun verhalten sich Löslichkeit und Löslichkeitsabnahme bei den Uraten annähernd so:

1) Zeitschr. f. physik. Chem., Bd. XXXIV, S. 435.

) Ibid., Bd. XXXVII, S. 385; Bd. XLVII, S. 357. 


\begin{tabular}{ll|c|c}
\hline Urat & Löslichkeit & $\begin{array}{c}\text { Löslichkeits- } \\
\text { abnahme }\end{array}$ \\
\hline $18^{\circ}$. & Kaliumurat & $1: 653$ & $8,4 \%$ \\
& Natriumurat & $1: 1201$ & $7,8 \%$ \\
& Ammoniumurat & $1: 2415$ & $31,0 \%$ \\
$37^{\circ}$. & $1: 370$ & $7,0 \%$ \\
& Kaliumurat & $1: 665$ & $4,7 \%$ \\
& Ammoniumurat & $1: 1352$ & $31,7 \%$
\end{tabular}

Das am schwersten lösliche Ammoniumurat hat zwar die größte Löslichkeitsabnahme, die beiden andern Urate verhalten sich aber gerade umgekehrt; das ist um so auffallender, als der Unterschied in der Löslichkeit fast $100 \%$ beträgt.

F. Kohlrausch ${ }^{1}$ ) hat überhaupt den Einfluß der Korngröße auf die Löslichkeit geleugnet. Wenn nun auch nach den Arbeiten von Hulett dessen Ansicht nicht mehr haltbar ist, so erscheint es mir bei einem Vergleich zwischen meinen Versuchsanordnungen und denen Huletts unstatthaft, beim Ammoniumurat diese weitgehende Löslichkeitsabnahme einfach mit der Veränderung der Korngröße erklären zu wollen. Hulett hat außer verschiedenen anderen Maßnahmen feinste Technik bei Pulverisierung der Substanz angewandt, ich habe nur darauf geachtet, daß die Salze einigermaßen gut gepulvert waren. Nach Rothmund ${ }^{2}$ ) ist aber nur dann eine anormale Löslichkeit zu erwarten, wenn die zu untersuchende Substanz außergewöhnlich fein ist. Während die obere Grenze bei Gips $2 \mu$ betrug, war nach meinen Messungen die Größe der Uratkrystalle sicherlich größer als $2 \mu$.

Nach Huletts Angaben war die Größe der Gipskrystalle in der neuntägigen Versuchsdauer von $0,3 \mu$ auf $2 \mu$ im Durchmesser, also ums Siebenfache, gewachsen. Ich versuchte die Größe der Uratkrystalle zu messen. Indessen haben diese Nadelform und sind schon in dem Zustand, in dem sie dem Lösungswasser zugefügt werden, von so verschiedener Dicke

1) Zeitschr. f. physik. Chemie, Bd. XLIV, S. 199.

2) Handbuch der angewandten Chemie, Bd. VI, S. 109, 1907. 
und Länge, daß ein Mittelwert, selbst aus Zählung sehr zahlreicher Individuen gewonnen, nur sehr beschränkten Wert hat. Zudem zerbrechen die Nadeln beim Filtrieren und beim Pulvern des Trockenrückstandes, sodaß aus diesen auf die ursprüngliche Korngröße in der Flüssigkeit nicht mehr rückgeschlossen werden darf.

Auch Versuche, die Krystallindividuen, in oestimmten Gewichtsverhältnissen aufgeschwemmt, in einer Zeissschen Zählkammer zu zählen, haben zu keinem sicheren Ergebnis geführt.

Wenn nach alledem ein gewisser Einfluß der Korngröße auf die Löslichkeitsabnahme zuzugeben ist, so lassen anderseits die verschiedenen angeführten Tatsachen und Überlegungen eine eindeutige Erklärung der Löslichkeitsabnahme in diesem Sinne zunächst nicht zu.

Schließlich wäre noch an eine Veränderung der Natur des Lösungsmittels $\mathrm{zu}$ denken. Nach Roth, ${ }^{1}$ ) Rothmund ${ }^{2}$ ) und $\mathrm{Biltz}{ }^{3}$ ) wird die lösende Wirkung des Wassers merklich durch Zusatz von Elektrolyten, besonders solcher mit mehrwertigen Ionen, verringert. Nernst ${ }^{4}$ ) führt diesen Einfluß auf die Veränderung der Dichte des Wassers zurück. Da aber derartige Veränderungen sehr viel schneller vor sich gehen müssen, als sich die beobachtete Löslichkeitsabnahme bei den Uraten vollzieht, ist auch diese Deutung nicht angängig.

So muß die festgestellte Löslichkeitsabnahme bei den drei Uraten vorläufig unaufgeklärt bleiben.

In späteren Untersuchungen soll dieser interessanten Erscheinung aber weiter nachgegangen werden.

Die gefundenen höchsten Löslichkeiten des primären Kalium-, Natrium- und Ammoniumurats bei $18^{\circ}$ und $37^{\circ}$.

Zur Ermittelung der betreffenden Löslichkeiten sind nur die Versuchsergebnisse herangezogen worden, welche als unbedingt zuverlässig betrachtet werden konnten. Die Abweichungen der

1) Zeitschr. f. physik. Ghem., Bd. XXIV, S. 114.

2) Ibid., Bd. XXXIII, S. 401.

s) Ibid., Bd. XLIII, S. 41.

4) Theoretische Chemie, S. 382. 
einzelnen Versuchsergebnisse sind auf Seite 163 näher definiert und als innerhalb der Fehlergrenzen liegend anzusehen.

Zur Ermittelung der Löslichkeitswerte bei $37^{\circ}$ sind auch Versuche verwertet worden, bei denen die Salze etwas länger als 15 Minuten, dem Sättigungszeitpunkt, rotiert haben, um Fehler auszuschließen, die sich durch etwaige Übersättigung hätten ergeben können, wenn auch eine solche, nach den Versuchsergebnissen zu urteilen, nicht bestanden zu haben scheint. ${ }^{1}$ )

Woher beim Natriumurat die Unterschiede zwischen den von $\mathrm{His}$ und $\mathrm{Paul}$ (S. 53) und von mir gewonnenen Werten herrühren, läßt sich zunächst noch nicht angeben. Zwar war die Bereitung der Salze verschieden; Nr. II a und II b des Versuchs von His und Paul sind mit einem Natriumurat angestellt, das auf kaltem Wege gewonnen war durch Auflösen der Harnsäure in überschüssiger Natronlauge und langsamem Überleiten von Kohlensäure; Nr. III a und b mit einem Präparat, das aus amorphem Urat durch Umkrystallisieren in heißer Natriumchloridlösung gewonnen war. Indessen war jede dieser Fraktionen auf ihren Harnsäuregehalt geprüft worden und hatte den für $\mathrm{C}_{5} \mathrm{H}_{3} \mathrm{~N}_{4} \mathrm{O}_{3} \mathrm{Na}+\mathrm{H}_{2} \mathrm{O}$ berechneten Wert ergeben.

Die Werte für das Kalisalz stimmen innerhalb der Fehlergrenzen mit den meinigen überein; für das Ammoniumsalz ist die von His und $\mathrm{Paul}$ gefundene Löslichkeit geringer, woran wahrscheinlich die lange Berührungsdauer von Salz und Lösung schuld ist, sodaß His und Paul nicht den maximalen Löslichkeitswert erhalten haben.

\section{Zusammenfassung der Untersuchungsergebnisse.}

1. In wässerigen Lösungen sind nur die primären harnsauren Salze beständig. Die Angaben in der Literatur über die Konzentration sekundärer harnsaurer Salze in wässerigen Lösungen beruhen also auf einem grundsätzlichen Irrtum.

2. Das primäre harnsaure Natrium hat auf $1 \mathrm{Mol} \mathrm{Salz}$ $1 \mathrm{Mol} \mathrm{H}_{2} \mathrm{O}$-Krystallwasser, das primäre harnsaure Kalium und Ammonium sind krystallwasserfrei.

1) Die Löslichkeitswerte sind auf nebenstehender Tabelle zusammengestellt. 
Über das Verhalten der harnsauren Salze in Lösungen.

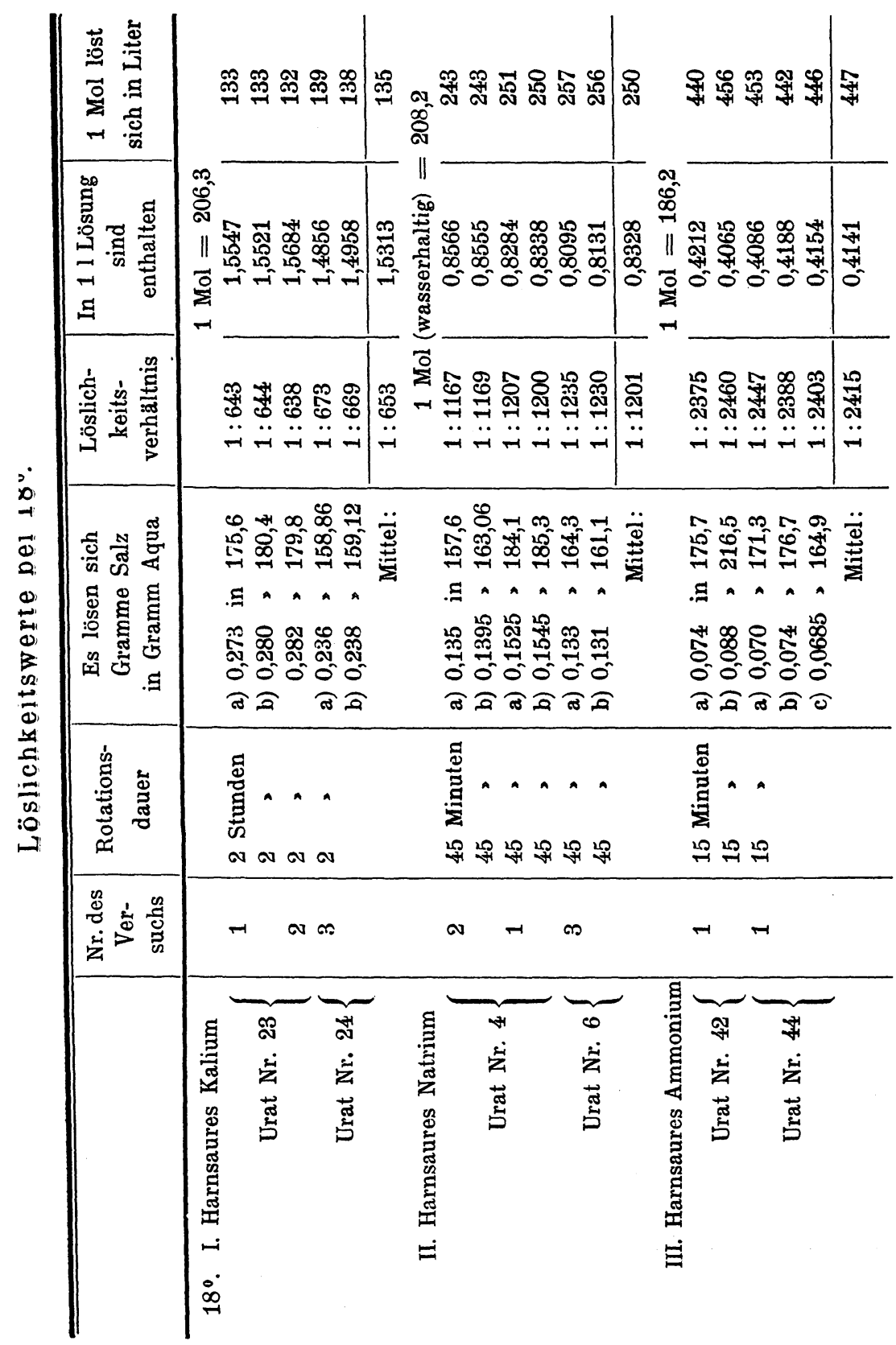




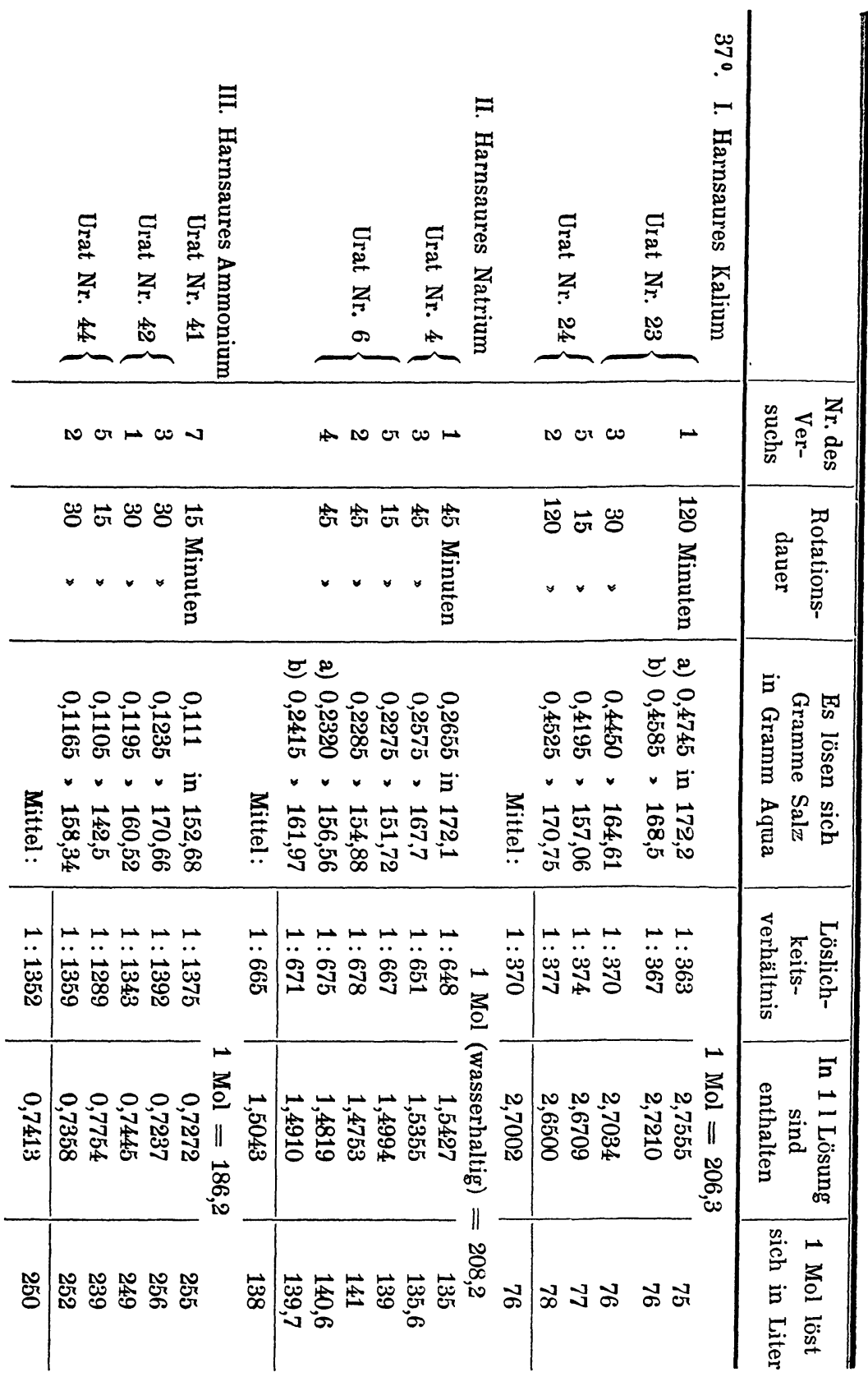


3. Alle Salze, am leichtesten das Ammoniumurat, zersetzen sich beim Erwärmen über $60^{\circ}$, indem sie an der Oberfläche eine rötliche Färbung annehmen. Auch unterhalb $60^{\circ}$ bei längerer Erwärmung und selbst bei Zimmertemperatur innerhalb einiger Monate tritt die Zersetzung ebenfalls auf.

4. Es ergab sich die bemerkenswerte Tatsache, daß die Löslichkeit der Salze nach Erreichung des Sättigungspunktes allmählich wieder abnimmt und die Geschwindigkeit dieser $\mathrm{Ab}$ nahme immer geringer wird, je länger man das Salz schüttelt. Es besteht also mit größter Wahrscheinlichkeit neben einem Lösungsmaximum unter den gleichen äußeren Bedingungen ein Lösungsminimum.

Die Tendenz zur Löslichkeitsabnahme ist sowohl bei $18^{\circ}$ wie bei $37^{\circ}$ beim Kalium- und Natriumurat annähernd gleich groß, beim Ammoniumurat aber 4-5mal größer.

Die Änderung der Temperatur von $18^{\circ}$ auf $37^{\circ}$ hatte auf diese Tendenzen keinen Einfluß.

Die Ursachen dieser Löslichkeitsabnahme konnten bisher nicht mit Sicherheit aufgefunden werden.

5. Die gefundene höchste Löslichkeit der drei Urate, deren Sättigungspunkt sowie deren Hydrolysengrad hat folgende Werte (siehe Tab. S. 178).

\section{Literaturverzeichnis.}

1. His und $\mathrm{Paul}$, Physikalisch-chemische Untersuchungen über das Verhalten der Harnsäure und ihrer Salze in Lösungen (Diese Zeitschrift, Bd. XXXI, 1900, und Pharmaz. Zeitung, 1900).

2. Bensch, All an und Baumgarten, Harnsaure Salze (Liebigs Annalen der Chemie und Pharmacie, Bd. LIV, S. 183; Bd. LXV, S. 181, und Bd. GXVII, S. 106).

3. Maly, Jahresbericht der Chemie (1863, S. 621).

4. Ostwald, Über die vermeintliche Isomerie des roten und gelben Quecksilberoxyds und die Oberflächenspannung fester Körper (Zeitschrift f. physik. Chemie, Bd. XXXV, S. 435).

5. Hulett, Beziehungen zwischen Oberflächenspannung und Löslichkeit; Löslichkeit und Korngröße (Zeitschrift f. physik. Chemie, Bd. XXXVII, S. 385, und Bd. XLVII, S. 357).

6. F. Kohlrausch, Über gesättigte wässerige Lösungen schwer löslicher Salze (Zeitschr. f. physik. Chem., Bd. XLIV, S. 199). 
F. Gudzent,

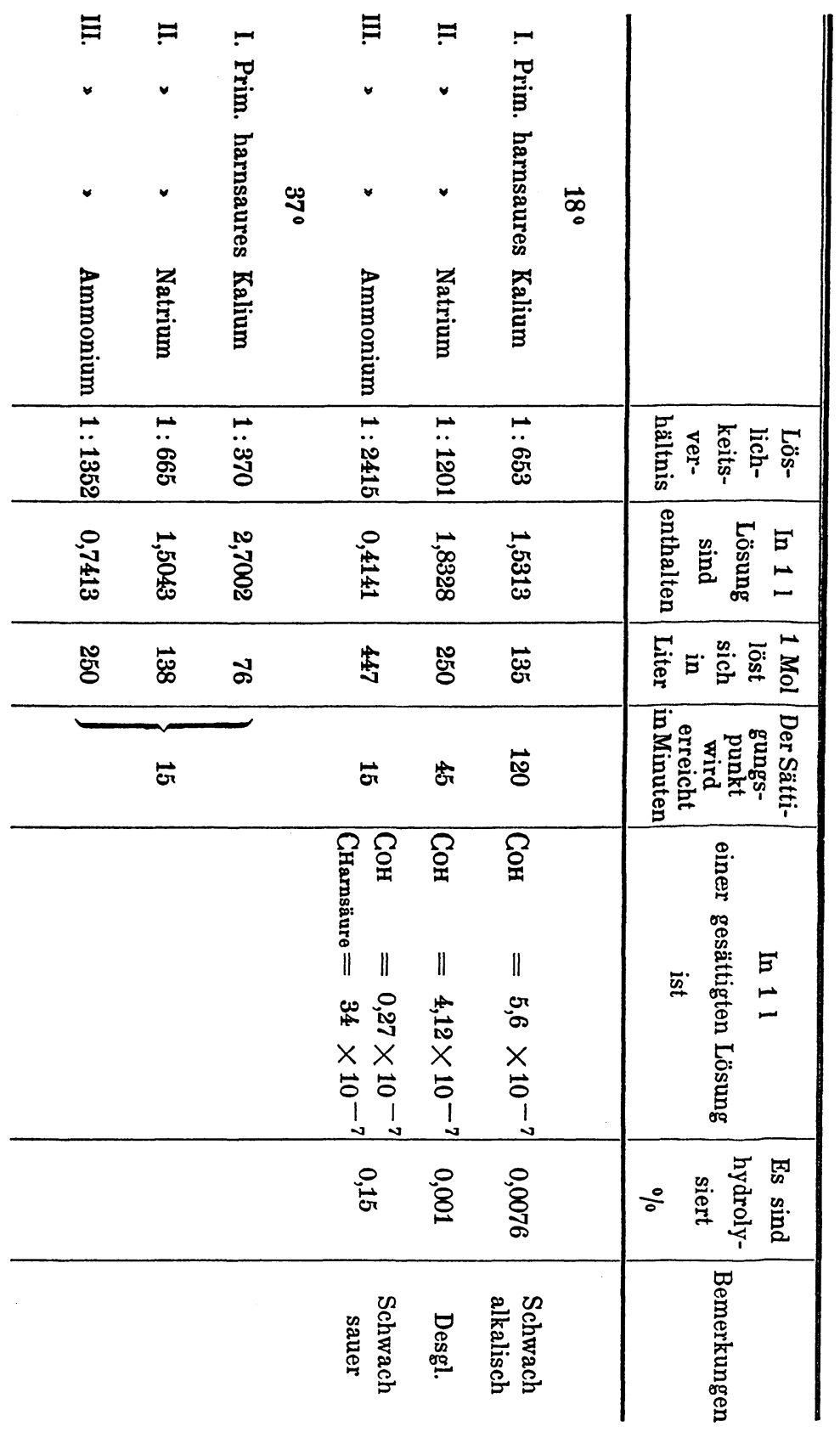


7. Abegg, Die Theorien der elektrolytischen Dissoziation. Handbuch der anorganischen Chemie, Bd. III, 3, S. 74, 1907.

8. Höber, Physikalische Chemie der Zelle und Gewebe, 2. A., 1906.

9. Ostwald, Lehrbuch der allgem. Chemie, 2. A., 1903.

10. Nernst, Theoretische Chemie, 5. A., 1907.

11. Traube, Grundriß der physik. Chemie, 1904.

12. Rothmund, Löslichkeit und Löslichkeitsbeeinflussung, 1907.

13. Beilstein, Handbuch der organischen Chemie, 3. A., 1903.

14. Ost wald-Luther, Hand- und Hilfsbuch zur Ausführung physikochemischer Messungen, 2. A., 1902.

15. Sir W. Roberts, Uric Acid. Gravel and Gout, London 1892.

16. Van't Hoff, Vorlesung über Bildung und Spaltung von Doppelsalzen, S. 125 u. 130.

Die Untersuchungen sind im Laboratorium der medizinischen Klinik in Göttingen unter Leitung des Herrn Geheimrat Professor Dr. med. His im Wintersemester 1906/07 und Sommersemester 1907 ausgeführt worden. 